\title{
Optimal demand response for thermal inertial loads employing stochastic renewables: A privacy respecting architecture and its continuum scaling limit
}

\author{
Gaurav Sharma ${ }^{1}$, Le Xie ${ }^{1}$ and P. R. Kumar ${ }^{1}$
}

\begin{abstract}
We consider the problem of optimally utilizing intermittently available renewable energy sources for inertial thermal loads. The particular focus of this paper is to reduce the need for dispatchable fossil fuel generation backup reserves, while satisfying thermal constraints of the loads, and to do so within an architecture where the individual loads control their own demands in a distributed manner so that privacy of every load's thermal state is respected. In this distributed architecture, no information about load states is communicated to the load aggregator. We model th e system as a distributed stochastic control problem with a control law parametrized by the individual set points of the loads, where the stochastic uncertainty is induced both by renewable energy availability as well as user temperature constraints. We design, optimize and analyze the parametrized policy, which has the key properties that it is privacy-respecting, employs distributed control via individual set-points, has low communication requirements, and is architecturally simple. While the optimal policy for a finite number of loads is intractable, and increasingly so as the number of loads increases, we show that the infinite population scaling limit as the number of loads increases is tractable. We employ Pontryagin's Minimum Principle to evaluate this, and show that it provides a near-optimal solution for the finite case.
\end{abstract}

\section{INTRODUCTION}

Stochastic renewable power sources like wind can be preferentially utilized over other fossil fuel sources to cool inertial thermal loads. The resulting demand response scheme is not only beneficial to the environment, but also is cost-effective. Moreover the flexibility of the power requirements from thermal loads can be used to absorb the fluctuations in the intermittent power sources.

We consider the scenario where thermal loads are airconditioners having a desired range of comfortable temperatures. The loads can change this range on occasions. Except for the transient period when such a change is made, the demand response scheme is required to respect the imposed temperature limits. Due to such stringent requirement in adhering to the temperature range, during the periods when wind power is not available it becomes inevitable to supplement wind power with fossil fuel generation sources. This raises an important issue. One would like to reduce the fossil fuel based backup reserves needed to supplement

\footnotetext{
${ }^{1}$ Department of Electrical and Computer Engineering, Texas A \& $\mathrm{M}$ University. Email:\{gash, le.xie,prk\}@tamu.edu .

${ }^{2}$ This material is based upon work partially supported by NSF under Contract Nos. CPS-1239116, Science \& Technology Center Grant CCF0939370, and CCF-1331863, and by Department of Energy Bonneville Power Administration under Cooperative Agreement \#63077.
}

the intermittent availability of the wind. Thus one of our objectives is to reduce the variation in the dispatchable fossil fuel generation.

Another issue is that loads may change their temperature range simultaneously. This arises, for example, when all homes on Superbowl Sunday reduce their temperature range in their living rooms at the same time. When such synchronized changes occur, one would not like to incur huge peak power drawn from fossil fuel sources. This can be mitigated if all the homes are not "synchronized" in their wind power consumption, i.e., all homes do not behave identically. Thus, "symmetry breaking" is an important aspect of the solution. We have found in our previous work [1], [2], that when one allows in the system model for simultaneous stochastic changes in temperature settings by users, and further specifically employs a cost function penalizing variability in dispatchable fossil fuel generation drawn, then indeed there is symmetry breaking in the optimal solution. This is interestingly related to local concavity of the cost-to-go function.

As is well known in other contexts, e.g., queueing theory, there is great benefit in "resource pooling." In our context, this corresponds to bundling a large number of loads together since one can then smooth out their fossil fuel generation consumption, as noted above. For this purpose we employ an architecture that consists of a load aggregator (also called a "load serving entity" or simply an "aggregator").

An important concern at the load end that arises in the context of demand response is that of privacy of individual users. Individual loads do not want to reveal their thermal states to the aggregator. Thus we seek a solution where instantaneous temperature measurements of the loads are not required to be communicated to the aggregator. Moreover our goal is to have a solution where the aggregator does not need to send continuous actuation signals to each of the loads. This would require great communication bandwidth aside from being undesirable due to several other considerations such as link reliability issues. Thus we do not seek a centralized solution where the aggregator controls the cooling of each load. Rather we seek a solution where each load controls its own cooling in a distributed fashion.

To understand our approach in a simple context, consider a collection of several identical homes with, say, air-conditioners, each home specifying a comfort range $\left[\Theta_{\min }, \Theta_{\max }\right]$, within which its temperature should lie. Suppose local renewable generation in a standalone microgrid 
environment, say wind, is used to cool all homes till $\Theta_{\min }$, whenever it is blowing. Power from fossil fuels in the same microgrid is used only when the temperature hits $\Theta_{\max }$. Under such a policy, after a transient period, the airconditioners in the homes all turn on or off at the same time. This "temperature synchronized" behavior is undesirable. If some external event triggers all the homes to dial down their $\Theta_{\max }$ at the same time, then the resulting peak to average draw of power from fossil fuel is large.

To avoid this, we analyze a policy of staggering thermostat settings within the comfort ranges to minimize a measure of peak-to-average demand. We show that the optimal staggering can be relatively easily computed when the number of homes is large. Importantly, this scheme allows each load's cooling to be controlled by its own thermostat. It does not entail any flow of information in the reverse direction from a load, thus preserving privacy of loads.

\section{A. Related work}

There has been much research into utilizing inertial thermal loads for smart grid based demand response schemes. Some works, $\mathrm{Xu}$ et. al. [3], Short et. al. [4] have focused on improving the stability of the power system by a TCLbased ancillary service. Other works, Lu et. al. [5], [6] have used a price-based signal to defer thermal loads in order to reduce energy cost and to avoid peak power consumption. Some recent works, Ilic et. al. [7], Papavasiliou et. al. [8] have considered using thermal loads to assist integration of intermittent renewable energy sources.

Callaway et. al [9], [10] have proposed distributed direct load control based schemes to deliver load following and regulation in the grid. Kundu et.al. [11], [12] have considered set-point based policy to safely control response of large population of thermal loads.

This paper is an extension of our previous works [1], [2], which considers controlling thermal loads in order to maximize renewable power integration, while reducing variations in the dispatchable fossil fuel generation requirement. Specifically, we extend the results of [2] by adding a preemptive constraint to adhere to stochastic comfort range requirements. This addition ensures that the temperature constraints set by the loads are met at all times, except when that is made unavoidable by the loads suddenly changing their comfort range to exclude the temperature that they are currently at. In that case, our solution violates the constraint for the shortest possible time that is required in order for the temperature to return to the comfort range.

\section{ARChiteCtURE, SET-POINT POLICY, WIND AND COMFORT RANGE VARIATION MODEL}

We consider an architecture consisting of an Aggregator, with the loads choosing the comfort range. The Aggregator observes the wind process, and transmits a signal to indicate its availability as necessary. This is akin to a "price-signal." Each load has a thermostat fed additionally with this wind availability signal. Upon receiving such a common signal, the loads turn on their air-conditioning if their temperature is above the lower limit of the comfort range, and maintain it there otherwise. If the temperature of the load hits the upper limit of the comfort range (or the set-point), but there is no wind availability signal, then the load draws just enough power (necessarily from fossil fuel sources) to maintain it at that temperature.

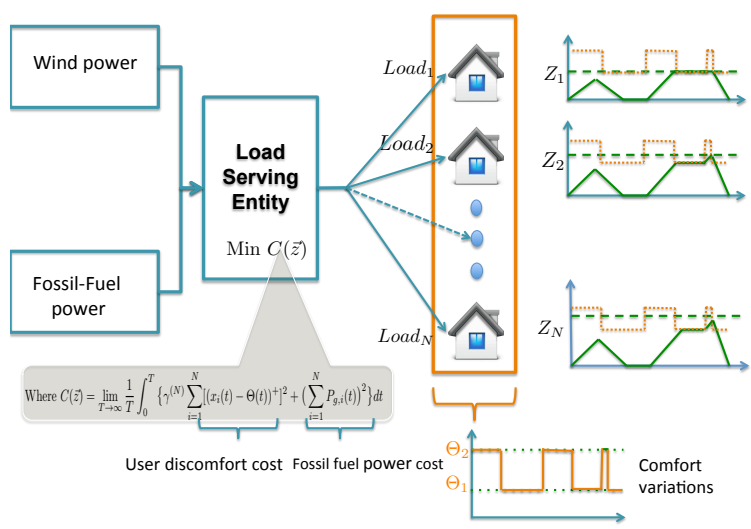

Fig. 1. The load serving entity provides a signal on availability of wind power to the loads, which each employ a thermostatic control policy. They only utilize wind power unless it drive the temperature above an upper Zlimit set by the policy. The cooling is reduced when the temperature hits the lower limit to not go below it.

We begin with a simple model of user load dynamics and comfort-range. Consider a collection of identical cooling loads, The temperature of load $i$ satisfies

$$
\dot{x}_{i}=h-P_{i},
$$

where $h$ is the effect of the ambient heating, and $P$ is the cooling power. The maximum cooling power is limited to $h+c$, so that the fastest rate of cooling yields $\dot{x}_{i}=-c$.

Every load has the desired comfort range $\left[0, \Theta_{M}(t)\right]$. To model simultaneous comfort zone changes, we suppose that all the loads occasionally change their common $\Theta_{M}(t)$ in a coordinated fashion at the same instant of time. For ease of exposition, we consider a simplified model. We suppose $\Theta_{M}(t)$ switches between two values $\Theta_{1}$ and $\Theta_{2}$ (with $\Theta_{1}<$ $\Theta_{2}$ ) as a Markov process with mean holding times $\frac{1}{r_{0}}$ and $\frac{1}{r_{1}}$, respectively. For each load $i$, we will further choose a value $Z_{i} \in\left[0, \Theta_{2}\right]$.

We model wind power as a binary Markov process, again for simplicity, with states "Blowing" and "Not Blowing", with mean holding times $\frac{1}{q_{1}}$ and $\frac{1}{q_{0}}$. When the wind is blowing we assume that it is sufficient to cool all the loads at the maximum rate.

When the wind is blowing, each load uses wind power to cool at the maximum rate $c$, unless it is already at its lowest permissible level in the comfort zone ( 0 in our case), in which case it uses just enough wind power to maintain itself at this lowest temperature. Thus

$$
\dot{x}_{i}(t)=\left\{\begin{array}{ll}
-c & \text { when } x_{i}(t)>0 \text { and wind is blowing } \\
0 & \text { when } x_{i}(t)=0 \text { and wind is blowing }
\end{array} .\right.
$$

When wind is not blowing, and, load- $i$ 's temperature either hits the upper limit of the comfort zone setting at that time instant or the value $Z_{i}$, the dispatchable fossil fuel generation is drawn to keep the temperature at that limit. If the comfort zone is ever changed in such a way that the current temperature is above its new upper limit, then 
maximum cooling is employed to return it as quickly as possible to the comfort zone. Hence, we obtain,

$$
\dot{x}_{i}(t)=\left\{\begin{array}{ll}
0 & \text { if } x_{i}(t)=\min \left(Z_{i}, \Theta_{M}(t)\right) \text { and wind is not blowing } \\
h & \text { if } x_{i}(t)<Z_{i} \text { and wind is not blowing } \\
-c & \text { if } x_{i}(t)>\Theta_{M}(t) \text { unconditionally }
\end{array} .\right.
$$

Denoting by $P_{w, i}(t)$ the power from wind, and by $P_{g, i}(t)$ the power drawn from fossil fuel sources, we have,

$$
\dot{x}_{i}(t)=h-P_{w, i}(t)-P_{g, i}(t),
$$

where,

and,

$$
P_{w, i}(t)=\left\{\begin{array}{ll}
h+c & \text { when } x_{i}(t)>0 \\
h & \text { when } x_{i}(t)=0
\end{array},\right.
$$

$$
P_{g, i}(t)=\left\{\begin{array}{ll}
h+c & \text { when } x_{i}(t)>\Theta_{M}(t) \& \text { wind not blowing } \\
h & \text { when } x_{i}(t)=\min \left(Z_{i}, \Theta_{M}(t)\right) \\
0 & \text { when } x_{i}(t)<Z_{i}
\end{array} .\right.
$$

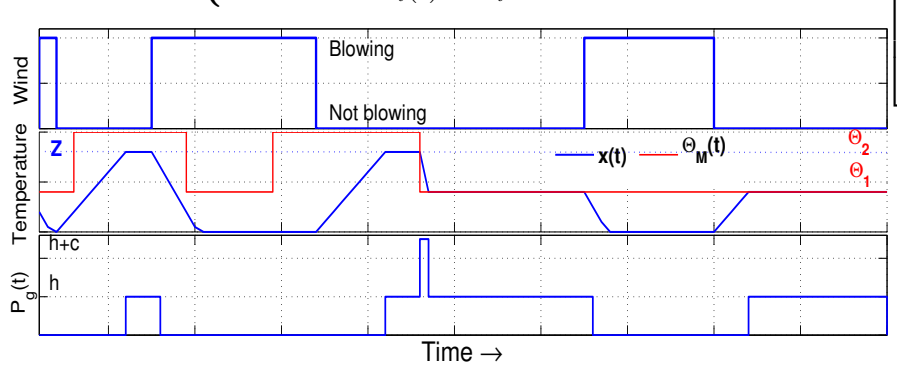

Fig. 2. The wind process, the temperature, and dispatchable fossil fuel generation drawn. Wind power is used whenever it is available to stay in comfort range. Fossil fuel power is used, if it must, to maintain the upper limit (or Z-value) to prevent comfort violation.

For $N$ loads, define $\mathbf{Z}:=\left(Z_{1}, Z_{2}, \ldots, Z_{N}\right)$, and let us call the above manner of operation as a "Z-Policy." When a user changes its comfort zone's upper temperature limit suddenly to be below its current temperature, then temporarily one has a violation of the comfort zone requirement. We ascribe a cost to this discomfort by $\left[\left(x_{i}(t)-\Theta(t)\right)^{+}\right]^{2}$ and consider the average cost of such discomfort $\lim _{T \rightarrow \infty} \frac{1}{T} \int_{0}^{T}\left[\left(x_{i}(t)-\right.\right.$ $\left.\Theta(t))^{+}\right]^{2} d t$

Since we want the total power drawn from fossil fuel sources $\sum_{i=1}^{N} P_{g, i}(t)$ at time $t$ to be as close to constant as possible, we impose a cost function $\lim _{T \rightarrow \infty} \frac{1}{T} \int_{0}^{T}\left[\sum_{i=1}^{N} P_{g, i}(t)\right]^{2} d t \quad$ to penalize "peak-to-average" power consumption. Any such choice serves to reduce variability and thus ensure a low backup reserve.

We therefore consider the following model illustrated in Figure 1 where the overall problem now consists of choosing $\mathbf{Z}=\left(Z_{1}, Z_{2}, \ldots, Z_{N}\right)$ to minimize the cost:

$$
\lim _{T \rightarrow \infty} \frac{1}{T} \int_{0}^{T}\left\{\gamma^{(N)} \sum_{i=1}^{N}\left[\left(x_{i}(t)-\Theta(t)\right)^{+}\right]^{2}+\left(\sum_{i=1}^{N} P_{g, i}(t)\right)^{2}\right\} d t
$$

where, $\gamma^{(N)}>0$ trades off discomfort against backup reserve.

\section{STEADY STATE DISTRIBUTION OF SET-POINT POLICY}

We begin by analyzing the steady state probability distribution resulting from a $Z$-policy employed in a single load. Let us focus on one of the thermostatically controlled loads. From Figure 2, it can be seen that the load will occupy each of temperature levels $x \in\left\{0, Z, \Theta_{1}\right\}$ for a non-zero fraction of time on average. Denote these steady state probability masses by $\delta_{0}^{Z}, \delta_{\Theta_{1}}^{Z}$, and $\delta_{Z}^{Z}$. For the intermediate temperatures $x \in\left(0, \Theta_{1}\right) \cup\left(\Theta_{1}, z\right)$, denote by $p_{i j}^{z}(x)$ for $i, j \in\{0,1\}$, the steady state occupation probability densities of the load at temperature $x$. Above, $i=0$ denotes wind off, $j=0$ denotes $\Theta_{M}(t)=$ $\Theta_{1}$, and $i=1$ denotes wind on, $j=1$ denotes $\Theta_{M}(t)=\Theta_{2}$. Let $\mathbf{p}^{\mathbf{z}}(x):=\left[\begin{array}{llll}p_{00}^{z}(x) & p_{01}^{z}(x) & p_{10}^{z}(x) & p_{11}^{z}(x)\end{array}\right]^{T}$.

Lemma 1: The probability distribution $\mathbf{p}^{z}(x), \delta_{0}^{z}, \delta_{\Theta_{1}}^{z}, \delta_{z}^{z}$ is given by the solution of the following linear system

$$
\frac{d \mathbf{p}^{z}(x)}{d x}=\left[\begin{array}{cccc}
-\frac{q_{0}+r_{0}}{k(x)} & \frac{r_{1}}{k(x)} & \frac{q_{1}}{k(x)} & 0 \\
\frac{q_{0}}{h} & -\frac{q_{0}+r 1}{h} & 0 & \frac{q_{1}}{h} \\
-\frac{q_{0}}{c} & 0 & \frac{q_{1}+r_{1}}{c} & -\frac{r_{1}}{c} \\
0 & -\frac{q_{0}}{c} & -\frac{r_{0}}{c} & \frac{q_{1}+r_{1}}{c}
\end{array}\right] \mathbf{p}^{z}(x) .
$$

where $k(x)=\left\{\begin{array}{ll}h & x<\Theta_{1} \\ -c & x>\Theta_{1}\end{array}\right.$. The boundary conditions are

$$
\left[\begin{array}{c}
\frac{h}{q_{0}+r_{0}} \\
0 \\
0 \\
0
\end{array}\right.
$$$$
\left[\begin{array}{cccc}
h / q_{1} & h / q_{1} & 0 & 0 \\
0 & 0 & c / q_{1} & c / q 1
\end{array}\right] \mathbf{p}^{z}(0)=\left[\begin{array}{l}
1 \\
1
\end{array}\right] \delta_{0},
$$$$
\left.\begin{array}{ccccccc}
0 & 0 & 0 & \frac{h}{q_{0}+r_{0}} & 0 & 0 & 0 \\
0 & 0 & 1 & 0 & 0 & 0 & -1 \\
-\frac{c}{q_{0}} & 0 & 0 & 0 & \frac{c}{q_{0}} & 0 & 0 \\
\frac{h}{r_{0}} & 0 & 0 & 0 & -\frac{h}{r_{0}} & 0 & 0
\end{array}\right]\left[\begin{array}{l}
\mathbf{p}^{z}\left(\Theta_{1}-\right) \\
\mathbf{p}^{z}\left(\Theta_{1}+\right)
\end{array}\right]=\left[\begin{array}{c}
\delta_{\Theta_{1}}^{z} \\
0 \\
\delta_{\Theta_{1}}^{z} \\
\delta_{\Theta_{1}}^{z}
\end{array}\right],
$$$$
\left[\begin{array}{cccc}
\frac{c}{r_{1}} & 0 & 0 & 0 \\
0 & \frac{h}{q_{0}+r_{1}} & 0 & 0 \\
0 & 0 & 1 & 0 \\
0 & 0 & 0 & \frac{c}{q_{0}}
\end{array}\right] \mathbf{p}^{z}(z)=\left[\begin{array}{l}
1 \\
1 \\
0 \\
1
\end{array}\right] \delta_{z}^{z},
$$$$
\int_{0}^{z}\left(\mathbf{1}^{T} \mathbf{p}^{z}(x)\right) d x+\delta_{0}^{z}+\delta_{z}^{z}+\delta_{\Theta_{1}}^{z}=1,
$$$$
\int_{0}^{z} p_{01}^{z}(x) d x+\delta_{z}^{z}=\frac{q_{1} r_{0}}{\left(q_{1}+q_{0}\right)\left(r_{1}+r_{0}\right)} .
$$

Proof: Equations (2-5) are Chapman-Kolmogorov equations, and (6-7) are marginalization of the probability distribution. Two linear differential equation in (2) entails 8 variables. This, along with 3 delta variables $\left(\delta_{0}^{z}, \delta_{\Theta_{1}}^{z}, \delta_{z}^{z}\right)$ are a linear system in 11 variables, with 11 linearly independent equations (3-7), which yields desired distribution. (There is a linear dependence in (3-7), since $\nu_{1}=[-h,-h, c, c]$ and $\nu_{2}=[c,-h, c, c]$, equations (3)-(5) gives $\nu_{1} \mathbf{p}^{z}(0)=0$, $\nu_{1} \mathbf{p}^{z}\left(\Theta_{1}-\right)=\nu_{2} \mathbf{p}^{z}\left(\Theta_{1}+\right)$ and $\nu_{2} \mathbf{p}^{z}(z)=0$.

Figure 3 illustrates a particular solution for the distribution function, which shows three delta functions at $0, \Theta_{1}, z$ and density functions for the corresponding four states of wind and upper comfort level setting. Such a solution exists for any number of comfort level settings.

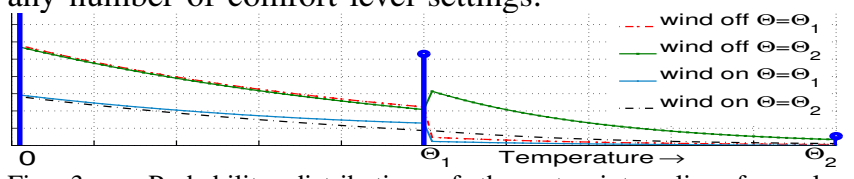

Fig. 3. Probability distribution of the set-point policy for values $\left(z, \Theta_{1}, \Theta_{2}, q_{0}, q_{1}, r_{0}, r_{1}, c, h\right)=(100,100,50, .04, .04, .02, .02,1.1,1)$. It consists of three point masses, and density functions which are continuous except at $\Theta_{1}$

\section{AnAlysis of the Finite loAds CASE}

Figure 4 shows typical temperature trajectories for the case when $N=3$, and $\mathbf{Z}=(60,70,80)$ with $\Theta_{2}=100$. Since we are considering the average cost problem, we can assume without loss of generality that all loads start with the same initial temperature. In order to calculate the average cost, we need the distribution of $\sum_{i=1}^{N} P_{g, i}$, which entails knowing the joint probability distribution of $\left\{P_{g, i}\right\}_{i=1}^{N}$. More generally, as we consider a large number of loads, which we do in the sequel, we need the joint distribution of a large number of loads' grid power draws. In order to determine this, an important property that we will exploit is that of stochastic domination. For any two loads $i$ and $j$ with $Z_{i}<Z_{j}$ we 
have $x_{i}(t) \leq x_{j}(t)$ for all $t$, regardless of the wind process realization. An important consequence is that whenever the $j$-th load hits its upper temperature limit $Z_{i}$, the $i$-th load has already hit its upper temperature limit too. Using $\omega$ to denote a sample point in the probability space, we have the following inclusion of events:

$$
\left\{X_{j}(t, \omega)=Z_{j}\right\} \subset\left\{X_{i}(t, \omega)=Z_{i}\right\}, \quad \text { if } Z_{i}<Z_{j} .
$$

Now we can calculate the expected cost of a $Z$-policy. First

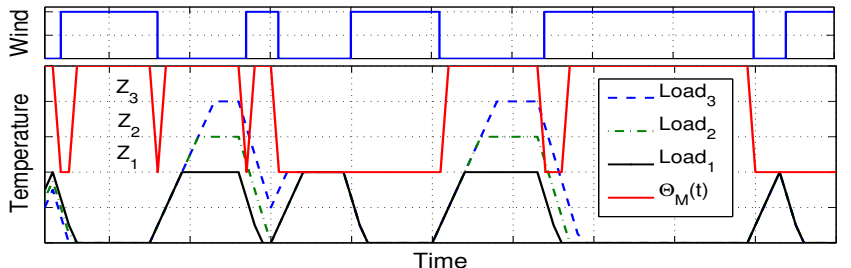

Fig. 4. When the wind is blowing, loads cool at the maximum rate, subject to not going below temperature 0, load temperatures do not rise above minimum of $Z$ and $\Theta_{M}(t)$

consider just one TCL. The fossil fuel power drawn is $h$ when $\Theta(t)=\Theta_{2}$ and the load temperature is at $Z$, while it is $h+c$ when $X(t)>\Theta(t)$. The cost due to discomfort is $\gamma^{(1)}\left[(x(t)-\Theta(t))^{+}\right]^{2}$, For any event $A$, denoting $\mathbb{P}^{i}(A):=$ $\mathbb{P}\left(A \cap\left\{\Theta(t)=\Theta_{i}\right\}\right)$ for $i=1,2$. The cost of the $Z$-policy is $C^{(1)}(Z)=h^{2} \mathbb{P}^{2}(X=Z)+\left[(h+c)^{2} \mathbb{P}^{1}\left(X>\Theta_{1}\right)+h^{2} \mathbb{P}^{1}\left(X=\Theta_{1}\right)\right]$ $+\gamma^{(1)} \int_{0}^{z}\left[\left(x-\Theta_{1}\right)^{+}\right]^{2}\left(p_{00}^{z}(x)+p_{10}^{z}(x)\right) d x$.

For brevity let us denote the second term by $\Phi(z)$,

$$
\Phi(z):=\int_{0}^{z}\left[\left(x-\Theta_{1}\right)^{+}\right]^{2}\left(p_{00}^{z}+p_{10}^{z}\right)(x) d x .
$$

Now we consider the case of two loads. In this case we need to consider the total dispatchable fossil fuel generation drawn by the two loads. Thus we need the joint probability distribution of the two loads. Their marginals will not suffice, unlike the case of just one load. It is here that we exploit the above stochastic dominance which provides information on the joint-distribution of the loads. When $\left(\Theta_{M}(t)=\Theta_{2}\right)$ with $Z_{1} \leq Z_{2}$, the total fossil fuel power is $2 h$ when $X_{2}=Z_{2}$ because $X_{2}=Z_{2} \Rightarrow X_{1}=Z_{1}$. Also, when $X_{2}<Z_{2}$ but $X_{1}=$ $Z_{1}$, the fossil fuel power is $h$. Similarly when $\Theta_{M}(t)=\Theta_{1}$, total fossil fuel power is $2(h+c)$ when $X_{1}>\Theta_{1},(h+c)+h$ when $X_{1}=\Theta_{1}$ but $X_{2}>\Theta_{2}$, and $2 h$ when $X_{2}=\Theta_{2}$. So the total cost $C^{(2)}$ with relative weight $\gamma^{(2)}>0$ is,

$$
\begin{aligned}
& C^{(2)}(\mathbf{Z})=(2 h)^{2} \mathbb{P}^{2}\left(X_{2}=Z_{2}\right)+h^{2} \mathbb{P}^{2}\left(X_{2}<Z_{2} \cap X_{1}=Z_{1}\right) \\
& \quad+(2 h)^{2} \mathbb{P}^{1}\left(X_{2}=\Theta_{1}\right)+(2(h+c))^{2} \mathbb{P}^{1}\left(X_{1}>\Theta_{1}\right) \\
& \quad+((h+c)+c)^{2} \mathbb{P}^{1}\left(X_{2}>\Theta_{1} \cap X_{1}=\Theta_{1}\right)+\gamma^{(2)}\left(\Phi\left(Z_{1}\right)+\Phi\left(Z_{2}\right)\right) .
\end{aligned}
$$

Also, since, $\left\{X_{2}=Z_{2}\right\} \subset\left\{X_{1}=Z_{1}\right\}$, we have $\mathbb{P}\left(\left\{X_{1}=Z_{1}\right\}\right)=$ $\mathbb{P}\left(\left\{X_{1}=Z_{1}\right\} \cap\left\{X_{2}<Z_{2}\right\}\right)+\mathbb{P}^{2}\left(\left\{X_{2}=Z_{2}\right\}\right)$, i.e.,

$$
\mathbb{P}^{2}\left(\left\{X_{1}=Z_{1}\right\} \cap\left\{X_{2}<Z_{2}\right\}\right)=\delta_{Z_{1}}^{Z_{1}}-\delta_{Z_{2}}^{Z_{2}} \text {. }
$$

When $\Theta(t)=\Theta_{1}$, by a similar argument we obtain,

$$
\mathbb{P}^{1}\left(\left\{X_{1}=\Theta_{1}\right\} \cap\left\{X_{2}>\Theta_{2}\right\}\right)=\delta_{\Theta_{1}}^{Z_{1}}-\delta_{\Theta_{1}}^{Z_{2}} .
$$

This analysis extends to the case of $N$ loads. For any choice $\left(Z_{1}, Z_{2}, \ldots, Z_{N}\right)$ of the set points with $Z_{1} \leq Z_{2} \ldots \leq Z_{N}$, we can evaluate the value of the average. It is

$C^{(N)}=N^{2}\left[h^{2}\left(\mathbb{P}^{2}\left(X_{1}=Z_{1}\right)+\mathbb{P}^{1}\left(X_{N}=\Theta_{1}\right)\right)+(h+c)^{2} \mathbb{P}^{1}\left(X_{1}>\Theta_{1}\right)\right]$

$+\gamma^{(N)} \sum_{k=1}^{N} \Phi\left(Z_{i}\right)+\sum_{k=1}^{N-1}\left[((N-k) h)^{2} \mathbb{P}^{2}\left(X_{k+1}=Z_{k+1} \cap X_{k}<Z_{k}\right)\right.$

$\left.+((N-k)(h+c)+(k h))^{2} \mathbb{P}^{1}\left(X_{k}=\Theta_{1} \cap X_{k+1}>\Theta_{1}\right)\right]$
Now we turn to an important issue concerning the choice of the scaling parameter $\gamma^{(N)} \cdot \int_{0}^{T}\left(\sum_{i=1}^{N} P_{g, i}\right)^{2} d t$ grows like $\Omega\left(N^{2}\right)$, but $\int_{0}^{T} \sum_{i=1}^{N}\left[\left(x_{i}(t)-\Theta(t)\right)^{+}\right]^{2} d t$ grows like $\Omega(N)$. We therefore scale $\gamma^{(N)}$ as $\gamma^{(N)}=\gamma \cdot N$. Let $\widehat{C}^{(N)}:=\frac{C^{(N)}}{N^{2}}$ denote the normalized cost. It evaluates to

$$
\begin{aligned}
& \widehat{C}^{(N)}=h^{2}\left\{\mathbb{P}^{2}\left(X_{1}=Z_{1}\right)+\mathbb{P}^{1}\left(X_{N}=\Theta_{1}\right)\right\}+(h+c)^{2} \mathbb{P}^{1}\left(X_{1}>\Theta_{1}\right) \\
& +\frac{\gamma}{N} \sum_{k=1}^{N} \Phi\left(Z_{i}\right)+\sum_{k=1}^{N-1}\left[\left(1-\frac{k}{N}\right)^{2} h^{2} \mathbb{P}^{2}\left(X_{k+1}=Z_{k+1} \cap X_{k}<Z_{k}\right)\right. \\
& \left.+\left(\left(1-\frac{k}{N}\right)(h+c)+\left(\frac{k}{N} h\right)\right)^{2} \mathbb{P}^{1}\left(X_{k}=\Theta_{1} \cap X_{k+1}>\Theta_{1}\right)\right]
\end{aligned}
$$

We thereby arrive at the following optimization problem for the case of a finite number $N$ of TCLs:

$$
\begin{aligned}
\text { Minimize } & \widehat{C}^{(N)}(\mathbf{Z}) \\
\text { s.t. } & Z_{i} \leq Z_{i+1} \text { for } i=1,2, \ldots, N-1, \\
& Z_{1} \geq 0, Z_{N} \leq \Theta_{2} .
\end{aligned}
$$

This problem is intractable because of the large number of constraints. Motivated by this we next examine its infinite load population limit.

\section{CONTINUUM Limit}

We now consider the infinite population limit where there is a continuum of loads. Let $u(z)$ denote the fraction of loads with set points no more than $z$. Let $\mathcal{U}$ denote the space of piecewise continuous increasing positive functions on $[0,1]$, noting that $u \in \mathcal{U}$. The resulting cost, following a similar analysis to (11), is

$$
\begin{aligned}
& C^{[0,1]}(u)=\gamma \int_{0}^{\Theta_{2}} \Phi(z) u^{\prime}(z) d z+h^{2}\left(\delta_{\Theta_{2}}^{\Theta_{2}}+\delta_{\Theta_{1}}^{\Theta_{1}}\right) \\
& \quad+\int_{0}^{\Theta_{2}}\left[(h u)^{2}(z) \mathbb{P}^{2}\left(\left\{X_{z}=z\right\} \cap\left\{X_{z+d z}<z+d z\right\}\right)\right) \\
& \left.+(h u(z)+(h+c)(1-u(z)))^{2} \mathbb{P}^{1}\left(\left\{X_{z}=\Theta_{1}\right\} \cap\left\{X_{z+d z}>\Theta_{1}\right\}\right)\right] .
\end{aligned}
$$

The first term is the cost of violation of upper temperature limit, while the second term is that due to variability in total dispatchable fossil fuel generation. Note that from (9),(10) $\mathbb{P}^{2}\left(\left\{X_{z}=z\right\} \cap\left\{X_{z+d z}<z+d z\right\}\right)=-\frac{d \delta_{z}^{z}}{d z} d z$ and $\mathbb{P}^{2}\left(\left\{X_{z}=\right.\right.$ $\left.\left.\Theta^{1}\right\} \cap\left\{X_{z+d z}>\Theta^{1}\right\}\right)=-\frac{d \delta_{\Theta}^{z}}{d z} d z$.

Define $D_{1}(z):=-\frac{d \delta_{z}^{z}}{d z}, D_{2}(z):=-\frac{d \delta_{\Theta_{1}}^{z}}{d z}$. The above can be simplified (as in [2]) to

$$
\begin{aligned}
C^{[0,1]}(u)=h^{2}\left(\delta_{\Theta_{2}}^{\Theta_{2}}+\delta_{\Theta_{1}}^{\Theta_{1}}\right)+ & {\left[\int _ { 0 } ^ { \Theta _ { 2 } } \left[\gamma u^{\prime}(z) \Phi(z)+(h u(z))^{2} D_{1}(z)\right.\right.} \\
& \left.\left.+((h+c)-c u(z))^{2} D_{2}(z)\right] d z\right] .
\end{aligned}
$$

Ignoring the first term that does not deoend on $u$, we obtain the following Calculus of Variations problem:

$\operatorname{Min} J[u]=\int_{0}^{\Theta_{2}}\left[(h u(z))^{2} D_{1}+(h+c-c u(z))^{2} D_{2}+\gamma \Phi(z) u^{\prime}(z)\right] d z$

s.t. $\quad u \in \mathcal{U}, u(0)=0, u\left(\Theta_{2}\right)=1$.

If one informally uses the Euler-Lagrange equation, one obtains the resulting solution $u_{E L}(z)=\frac{\gamma \Phi^{\prime}(z)+2 c(c+h) D_{2}(z)}{2\left(h^{2} D_{1}(z)+c^{2} D_{2}(z)\right)}$. This however need not be a positive increasing function or satisfy the boundary condition $u_{E L}(0)=0, u_{E L}\left(\Theta_{2}\right)=1$. Figure shows a particular solution where $u_{E L} \notin \mathcal{U}$ and $u_{E L}\left(\Theta_{2}\right) \neq 1$.

Therefore the Euler Lagrange solution is not admissible for all the scenarios. We consider next how to solve this problem using Pontryagin's Minimum Principle. 


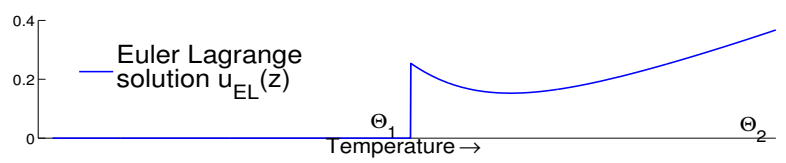

Fig. 5. The Euler Lagrange solution obtained for the values used to find distribution in Figure 3.

\section{OPTIMAL SOLUTION}

We employ the theory of optimal control to solve the optimization problem. Assume that state $u(z)$ follows the dynamic system $\dot{u}(z)=f(u, v, z)$ for any admissible control $v(z) \in \mathbf{V}$, and $z \in\left[0, \Theta_{2}\right]$. Consider the cost function is $J[u]=\int_{0}^{\Theta_{2}} g(u, v, z) d z$.

Defining $h(u, v, \lambda, z)=g(u, v, z)+\lambda(z) f(u, v, z)$, the optimal control $v^{*}(z)$ which takes the state $u(z)$ from $u(0)=0$ to $u\left(\Theta_{2}\right)=1$, satisfies the following two conditions [13]:

1) $\dot{\lambda}(z)=-\frac{\partial g}{\partial u}$

2) $v^{*}(z) \in \mathbf{V}$ is the pointwise minimizer of $h(u, v, \lambda, z)$.

Using Theorem 3.36 from [14], since $u(z) \in \mathcal{U}$ has bounded variation, and $\Phi(z)$ is continuous, we have

$$
\int_{0}^{\Theta_{2}} \Phi(z) u^{\prime}(z) d z=\Phi\left(\Theta_{2}\right)-\int_{0}^{\Theta_{2}} \Phi^{\prime}(z) u(z) d z .
$$

We use (13),(15) to collect terms depending on $u(z)$, to rewrite the cost in the following equivalent way:

$$
\begin{aligned}
J^{\prime}[u] & =\int_{0}^{\Theta_{2}} u^{2}(z) w(z)-u(z)\left(\gamma \Phi^{\prime}(z)+2 c(c+h) D_{2}(z)\right) d z \\
& =\int_{0}^{\Theta_{2}}\left[u(z)-u_{E L}(z)\right]^{2} w(z)-\frac{\left(\gamma \Phi^{\prime}(z)+2 c(c+1) D_{2}(z)\right)^{2}}{4\left(h^{2} D_{1}(z)+c^{2} D_{2}(z)\right)} d z
\end{aligned}
$$

Where $w(z):=\left(h^{2} D_{1}(z)+c^{2} D_{2}(z)\right)>0$. Thus, we will focus on optimizing the cost functional $J[u]=$ $\int_{0}^{\Theta_{2}} w(z)\left[u(z)-u_{E L}(z)\right]^{2} d t, u(0)=0$ and $u\left(\Theta_{2}\right)=1$. Wlog, we can take $u_{E L}(z) \in[0,1]$ (otherwise replace $u_{E L}$ by $\left.\max \left(0, \min \left(1, u_{E L}\right)\right)\right)$.

We first consider the Euler Lagrange solution $u_{E L}(z)$ obtained in Section IV. In the notations above, we identify $\mathbf{V}=\mathbb{R}, f(u, v, z)=v^{2}$ and $g(u, v, z)=w(z)\left[u(z)-u_{E L}(z)\right]^{2}$.

The optimizers $v^{*}, \lambda^{*}$ satisfy,

$$
\begin{aligned}
& \dot{\lambda}^{*}(z)=-2\left(u(z)-u_{E L}(z)\right) w(z) \\
& \dot{u}(z)=v^{* 2}(z) \\
& v^{*}(t)=\underset{v \in R}{\arg \min }\left[w(z)\left(u(z)-u_{E L}(z)\right)^{2}+\lambda^{*}(z) v^{2}(z)\right]
\end{aligned}
$$

One can rewrite (18) as:

$$
v^{*}(z)= \begin{cases}0 & \text { if } \lambda^{*}(z)>0 \\ -\infty & \text { if } \lambda^{*}(z)<0 \\ \text { arbitrary } & \text { if } \lambda^{*}(z)=0\end{cases}
$$

One can observe that $\lambda^{*}(z)$ can-not be strictly negative, as this leads to unbounded $u(z)$ from (17), and further results in infinite cost $J$. This also suggests that jumps in $u(z)$ are possible only at those points where $\lambda^{*}(z)$ is zero.

Also from (16), we observe that $\lambda^{*}(z)$ remains constant only on the points where $u(z)=u_{E L}(z)$, it increases when $u(z)<u_{E L}(z)$, and decreases when $u(z)>u_{E L}(z)$. From (17), (18) we observe that if $\lambda^{*}(z)>0$, then $v^{*}(z)$ is zero, leading to a constant $u(z)$. So, $\lambda^{*}(z)>0$ implies $u(z)=$ constant. Next, since $u(z)$ has to be increasing from (17), we conclude that $\lambda^{*}(z)$ can-not be constant on the points where $u_{E L}(z)$ is decreasing, however $\lambda^{*}(z)$ can be constant when $u_{E L}(z)$ is increasing.
Now we consider $u_{E L}(z)$ obtained in Section IV. Since we require $u^{*}\left(\Theta_{2}\right)=1$, there are jumps at $\Theta_{1}$ and $\Theta_{2}$. Next if $u_{E L}(z)$ is decreasing in $\left[\Theta_{1}, \theta\right]$ then $\lambda^{*}(z)$ is not constant in $\left[\Theta_{1}, \theta\right]$ and $u^{*}(z)$ is a constant in $\left[\Theta_{1}, \theta\right]$, let $\left.u^{*}\right|_{z \in\left[\Theta_{1}, \theta\right]}=\kappa$. Since $\lambda(z)$ can not be negative, $\kappa=u^{*}\left(\Theta_{1}\right)<$ $u_{E L}\left(\Theta_{1}\right)$ and also since $\lambda\left(\Theta_{2}\right)$ needs to be zero, $u\left(\Theta_{2}\right)>$ $\min _{z \in\left[\theta, \Theta_{2}\right]}\left\{u_{E L}(z)\right\}$, so that $\lambda(z)$ decreases when $u_{E L}(z)<\kappa$. $\lambda(z)$ decreases to zero, and subsequently stays at zero, and $u^{*}(z)$ remains equal to $u_{E L}(z)$ till $\Theta_{2}$. Note that $u^{*}(z)$ has a discontinuity at $\Theta_{2}$, since we require $u^{*}\left(\Theta_{2}\right)=1$. This is illustrated in Figure 6.

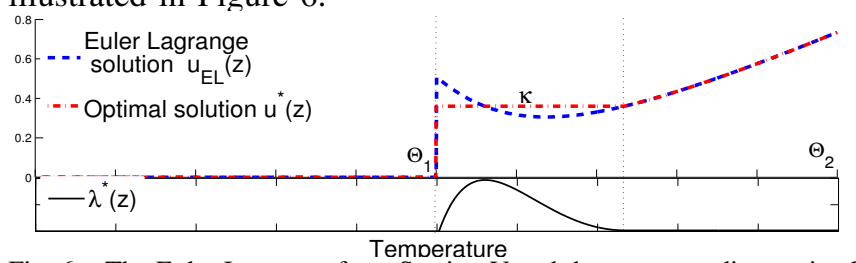

Fig. 6. The Euler Lagrange from Section V and the corresponding optimal solution obtained from the Pontryagin's minimum principle.

Notice that the above $\kappa$ satisfies $\int_{y_{1}}^{y_{2}}\left(\kappa-u_{E L}(z)\right) w(z) d z=0$, for $y_{1}:=\sup \left\{z<\theta: u_{E L}(z)<\kappa\right\}$ and $y_{2}:=\inf \{z>\theta$ : $\left.u_{E L}(z)>\kappa\right\}$. For a general $u_{E L}(z)$, we can find an optimal solution $u^{*}(z)$ in a similar way. We first find the partition $\left\{\Theta_{1}=\theta_{0}, \theta_{1}, \ldots, \theta_{N}=\Theta_{2}\right\}$, such that (wlog) $u_{E L}$ is increasing in $\left[\theta_{2 i}, \theta_{2 i+1}\right]$, and decreasing in $\left[\theta_{2 i-1}, \theta_{2 i}\right]$, and finding $\kappa_{i}$ such that $\int_{y_{2 i-1}}^{y_{2 i+i}}\left(\kappa_{i}-u_{E L}(z)\right) w(z) d z=0$, for $y_{2 i-1}=\sup \{z<$ $\left.\theta_{2 i}: u_{E L}(z)<\kappa_{i}\right\}$ and $y_{2 i+1}=\inf \left\{z>\theta_{2 i}: u_{E L}(z)>\kappa_{i}\right\}$. This is shown in Figure 7.

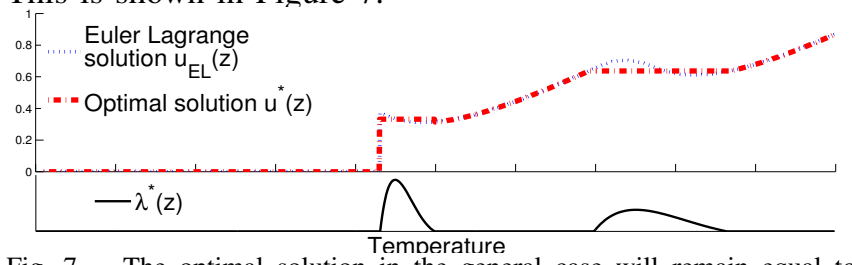

Fig. 7. The optimal solution in the general case will remain equal to Euler Lagrange solution in some intervals and will remain constant in other intervals.

\section{EXAMPLES AND SIMULATIONS}

\section{A. Comparison of finite and asymptotic cases}

We now compare the optimal asymptotic case with the numerically computed finite case optimal solution. Figure 8 shows that $u^{(N)}(z)$ and cost $C^{(N)}$ are remarkably close to $u^{*}(z)$ and $C^{*}$ respectively, even for the relatively small number of loads $N=5$.

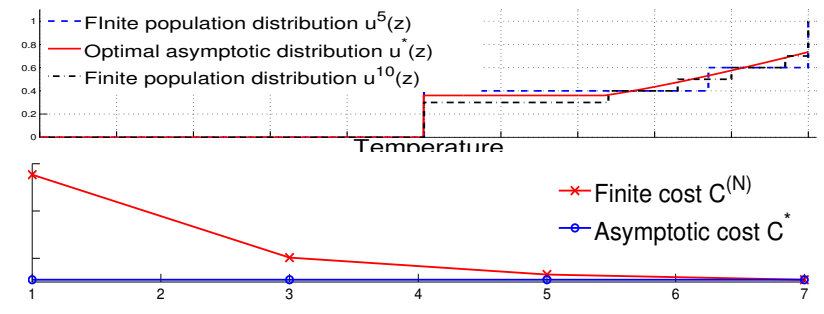

Fig. 8. Comparison of set-point distribution and cost for finite population optimization problem to the asymptotic cost, for parameters $\left(c, h, q_{0}, q_{1}, r_{0}, r_{1}, \gamma\right)=(1.1,1, .04, .04, .02, .02, .02)$.

\section{B. Cost of synchronization}

Figure 9 show that the optimal desynchronization results in a $25 \%$ lower dispatchable fossil fuel generation with negligible user discomfort, when compared to an optimal policy 
which maintains the same set-points. Figure 10 compares the load factor (Load Factor := $\frac{\text { Average Power }}{\text { Peak Power }}$ ), and illustrates the lower power fluctuations for the optimal desynchronized policy.

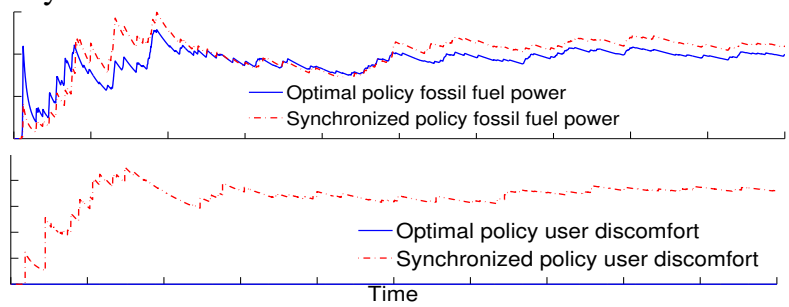

Fig. 9. Comparison of the average dispatchable fossil fuel generation and user discomfort between optimal synchronized policy and optimal staggered set-point policy, for population size $\mathrm{N}=10$.

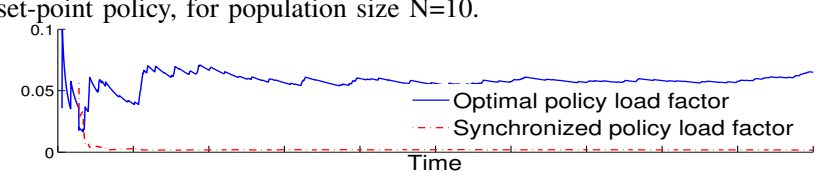

Fig. 10. Load factor comparison between the optimal synchronized policy and optimal staggered set-point policy, for population size $\mathrm{N}=10$.

\section{Effect of temperature variations in comfort range}

In the absence of comfort violation, clearly every load should set $Z \equiv \Theta_{2}$. However, if we employ such a policy when there are indeed comfort zone changes, the additional cost incurred can be large. When only a fraction of loads (chosen iid with $\mathrm{p}=0.5$ ), changes upper comfort level to $\Theta_{1}$. On the other hand, if we employ the optimal Z-policy which employs staggering in the latter case, we recover $290 \%$ of this increase. This is shown in Figure 11.

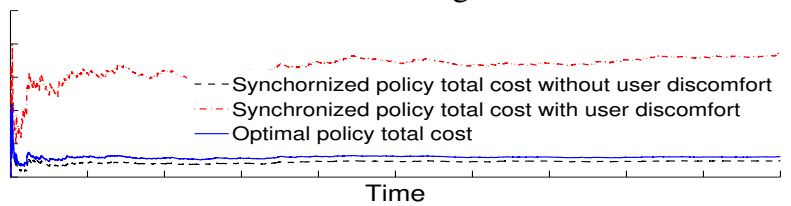

Fig. 11. Comparison of the total cost when a fraction of population changes their upper comfort level for population size $\mathrm{N}=10$.

\section{Approximation of solution to finite population by asymp-} totic solution

Figure 12 compares the $Z$-policy for the numerically computed optimal policy with the policy obtained by approximating by the infinite population policy as described earlier. Figure 13 compares the resulting time average dispatchable fossil fuel generation variation and temperature discomfort cost components. Apparently, in terms of cost, the approximate solution is a good substitute for the optimal solution.

\section{CONCLUDING REMARKS}

We have presented a holistic approach to the multidimensional aspects of the problem of utilization of wind power by inertial thermal loads. Our solution addresses several aspects: (i) the problem of reducing the variability that is imposed on the dispatchable fossil fuel generation to compensate for the intermittency of wind, and thus the problem of reducing backup reserve, (ii) the problem of ensuring robustness to sudden simultaneous changes in user comfort settings, (iii) the problem of protecting the privacy of users with respect to revealing their thermal states, (iv)

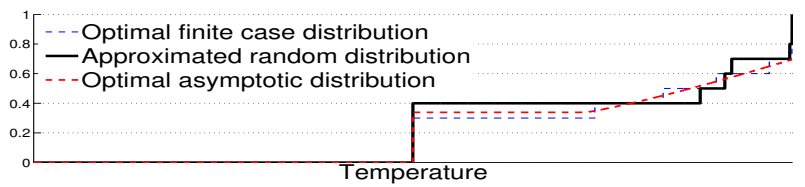

Fig. 12. Approximating the optimal distribution by randomly generating set-points according to the optimal asymptotic distribution.

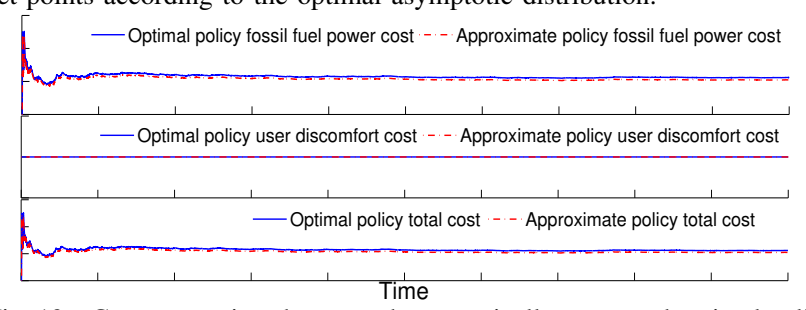

Fig. 13. Cost comparison between the numerically computed optimal policy and random approximated policy.

proposing a simple architecture that incorporates a "priceaware thermostat" as a simple implementation on the user end, (v) tractability of the solution procedure for both the cases of a large number of loads as well as a small number of loads. Our results can be generalized to allow more complex models of wind and loads.

\section{REFERENCES}

[1] G. Sharma, L. Xie, and P. R. Kumar, "On the optimality of desynchronized demand response with stochastic renewables and inertial thermal loads," in IEEE Conference on Decision and Control, pp. 6292-6297, Dec 2013.

[2] G. Sharma, L. Xie, and P. Kumar, "Large population optimal demand response for thermostatically controlled inertial loads," in Smart Grid Communications (SmartGridComm), 2013 IEEE International Conference on, p. 259, Oct. 2013.

[3] Z. Xu, J. Ostergaard, M. Togeby, and C. Marcus-Moller, "Design and modelling of thermostatically controlled loads as frequency controlled reserve," Power Engineering Society General Meeting, pp. 1-6, 2007.

[4] J. A. Short, D.G.Infield, and L. Freris, "Stabilization of grid frequency through dynamic demand control," IEEE Transactions on Power Systems, vol. 22, no. 3, pp. 1284-1293, 2007.

[5] N. Lu and S. Katipamula, "Control strategies of thermostatically controlled appliances in a competitive electricity market," IEEE Power and Energy Society General Meeting, vol. 1, pp. 202-207, 2005.

[6] N. Lu and D. P. Chassin, "A state-queueing model of thermostatically controlled appliances," IEEE Transactions on Power Systems, 2004.

[7] M. D. Ilic, L. Xie, and J.-Y. Joo, "Efficient coordination of wind power and price responsive demand-Part I: Theoretical foundations," IEEE Transactions on Power Systems, vol. 26, no. 4, pp. 1875-1884, 2011.

[8] P. Anthony and S. S. Oren, "Supplying renewable energy to deferrable loads: Algorithms and economic analysis," IEEE Power and Energy Society General Meeting, pp. 1-8, 2010.

[9] D. Callaway, "Tapping the energy storage potential in electric loads to deliver load following and regulation, with application to wind energy," Energy Conversion and Management, vol. 50, no. 9, pp. 1389-1400, 2009.

[10] D. Callaway and I. Hiskens, "Achieving controllability of electric loads," Proceedings of the IEEE, vol. 99, pp. 184-199, Jan 2011.

[11] S. Kundu, S. B. Nikolai Sinitsyn, and I. Hiskens, "Modelling and control of thermostatically controlled loads," in Power Systems Computation Conference, 2011.

[12] S. Kundu and N. Sinitsyn, "Safe protocol for controlling power consumption by a heterogeneous population of loads," in American Control Conference, 2012.

[13] L. S. Pontryagin, V. G. Boltyanskii, R. V. Gamkrelidze, and E. Mishchenko, The mathematical theory of optimal processes. Interscience publishers, 1962.

[14] G. B. Folland, Real analysis, Modern Techniques and Their Applications. Pure and Applied Mathematics: A Wiley Series of Texts, Monographs and Tracts, 1997. 